[Article]

\title{
氦液滴中羰基硫分子的光解动力学
}

\author{
张翠梅 ${ }^{1,2}$ 张志国 ${ }^{1,2}$ 黄存顺 ${ }^{2, *}$ 张 群 ${ }^{1, *}$ 陈 昒 ${ }^{1, *}$ \\ ('中国科学技术大学合肥微尺度物质科学国家实验室, 化学物理系, 合肥 230026; \\ 2 中国科学院大连化学物理研究所, 分子反应动力学国家重点实验室, 辽宁大连 116023)
}

\begin{abstract}
摘要: 用时间切片速度成像方法研究了氦液滴中羰基硫(COS)分子的光解动力学. 从共振增强 (2+1) 电离的 $\mathrm{CO}$ 光谱中发现在氦液滴环境中解离产物 $\mathrm{CO}$ 的转动冷却比振动冷却更有效. 利用速度成像采集到的 $\mathrm{CO}(\mathrm{v}=0)$ 和 $C O(v=1)$ 的影像在角分布上都呈现出各向同性的特点. 产物平动能分布的结果表明尽管大部分的平动能都 被超流体环境所驰豫, 但振动激发态产物 $\mathrm{CO}(v=1)$ 的平均平动能比振动基态产物 $\mathrm{CO}(v=0)$ 的平均平动能高. 对羰基硫分子在氦液滴中的光解动力学机理进行了讨论.
\end{abstract}

关键词: 氦液滴; 时间切片速度成像; 羰基硫 中图分类号: 0643

\section{Photodissociation Dynamics of Carbonyl Sulfide in Helium Droplets}

\author{
ZHANG Cui-Mei ${ }^{1,2}$ ZHANG Zhi-Guo ${ }^{1,2}$ HUANG Cun-Shun ${ }^{2, *}$ \\ ZHANG Qun ${ }^{1, *} \quad$ CHEN Yang ${ }^{1, *}$
}

('Hefei National Laboratory for Physical Sciences at the Microscale and Department of Chemical Physics, University of Science and Technology of China, Hefei 230026, P. R. China; ${ }^{2}$ State Key Laboratory of Molecular Reaction Dynamics, Dalian Institute of Chemical Physics, Chinese Academy of Sciences, Dalian 116023, Liaoning Province, P. R. China)

\begin{abstract}
The photodissociation dynamics of carbonyl sulfide (OCS) in helium droplets was studied using a velocity-map-imaging technique. The $\mathrm{CO}$ fragments were detected by $(2+1)$-resonance-enhanced multiphoton ionization. It was found that in the helium droplet environment, rotational cooling is much more efficient than vibrational cooling. The velocity map images for both $\mathrm{CO}(v=0)$ and $\mathrm{CO}(v=1)$ exhibit nearly isotropic angular distributions. The kinetic energy distributions show that most of the translational energies are relaxed in the finite-sized superfluid helium system. However, the average translational energies of the CO $(v=1)$ images are higher than those of the $C O(v=0)$ images. The relevant mechanism is briefly discussed.
\end{abstract}

Key Words: Helium droplet; Time-sliced ion imaging; OCS

\section{Introduction}

Helium droplets have been recognized as ideal cryogenic matrix isolation due to their ultralow temperature and superflu- idity.' One of the most remarkable properties is that the rotational temperature of molecules doped into droplets could be quickly and efficiently cooled to as low as $0.37 \mathrm{~K} .{ }^{2,3}$ Hence, the

Received: May 3, 2013; Revised: June 19, 2013; Published on Web: June 19, 2013.

"Corresponding authors. HUANG Cun-Shun, Email: cshuang@dicp.ac.cn; Tel: +86-411-84379775. ZHANG Qun, Email: qunzh@ustc.edu.cn; Tel: +86-551-3607736. CHEN Yang, Email: yangchen@ustc.edu.cn; Tel: +86-551-3606619.

The project was supported by the National Natural Science Foundation of China (21073187, 21273212, 21173205), National Key Basic Research Program of China (973) (2010CB923302), 100 Talents Program of Chinese Academy of Sciences, Knowledge Innovation Program of Chinese Academy of Sciences, and USTC-NSRL Joint Funds, China (KY2340000021).

国家自然科学基金项目 (21073187, 21273212, 21173205), 国家重点基础研究项目 (973)(2010CB923302), 中国科学院百人计划和中国科学院 知识创新项目, 中国科学技术大学-同步辐射联合基金 (KY2340000021)资助

(C) Editorial office of Acta Physico-Chimica Sinica 
spectra, structures, and dynamics of various isolated systems (such as atoms, molecular radicals, and ions) in helium droplets have been extensively studied over decades. ${ }^{4,5}$

The photodissociation dynamics of doped helium droplets drew attention of people recently. Braun and Drabbels ${ }^{6-8}$ studied the photodissociation of alkyl iodide molecules doped helium droplets with the ion imaging technique. Comparing with the gaseous phase results, the kinetic energy releases from the photodissociation of the doped helium droplets are much lower. A nonthermal "direct" mechanism that the photofragments push away surrounding helium atoms until they reach the droplets surface followed by escaping into the gaseous phase has been proposed. The classical Monte Carlo calculations based on a binary hard-sphere scattering model reproduced the experimental results very well. Besides the translational energy distribution, they observed some special phenomena such as fragment salvation dynamics and recombination process. These observations demonstrate that the finite-sized superfluid helium droplet system exhibits odd characteristic which affects the chemical reactions inside the droplets. Later, using the femtosecond pump-probe technique, Stapelfeldt et al. ${ }^{9}$ studied the alignment effect of the methyl iodide doped helium droplets system. The results show that the rotational dynamics of meth$\mathrm{yl}$ iodide dissolved inside the helium droplets is much slower than that of the isolated gaseoupus phase value. And the sharp transient alignment recurrence characteristic of gaseous phase molecules are absence in the doped droplets. All previous photochemistry studies on the doped helium droplets presage opportunities to explore both the chemical reaction dynamics inside the helium droplets and the properties of the finite-sized superfluid system.

As a benchmark system, carbonyl sulfide (OCS) has been vastly studied in the gas phase. ${ }^{10-18}$ Although there have been a number of reports on OCS molecules in helium droplets, they mainly concentrated on the spectroscopic aspects. ${ }^{2,19-21}$ For instance, the high resolution double resonance infrared-microwave $^{19}$ and the rotational spectra ${ }^{2}$ of a single OCS molecule in helium droplets have been obtained. To date, the photodissociation dynamics of OCS doped helium droplets are still obscure. As a complementary to the gaseous phase photodissociation dynamics, here we report on the photodissociation dynamics of OCS in helium nanodroplets by means of velocity map ion imaging (VMI) in combination with $(2+1)$ resonance enhanced multiphoton ionization (REMPI) detection. Firstly we report the CO REMPI from the OCS photodissociation. Then we obtain ion images of $\mathrm{CO}(v=0)$ and $\mathrm{CO}(v=1)$. The photodissociation dynamics of the OCS doped helim droplets have been discussed in light of the previous related works.

\section{Experimental}

The experiments were performed on a new constructed pulsed helium droplets-VMI apparatus. ${ }^{22}$ Briefly, the apparatus consists of three differentially pumped vacuum chambers named source, doping, and detection chambers. The source chamber is equipped with a large turbo molecular pump with the pumping speed of $2200 \mathrm{~L} \cdot \mathrm{s}^{-1}$ (STP-A2203C, Edwards, USA) to keep the pressure at $1 \times 10^{-6} \mathrm{~Pa}$ without the droplets beam and $\sim 2 \times 10^{-4} \mathrm{~Pa}$ with the beam. The doping chamber and detection chamber are equipped with nominal helium pumping speeds of $260 \mathrm{~L} \cdot \mathrm{s}^{-1}$ (TC600, Pfeiffer vacuum, USA) and $1000 \mathrm{l} \cdot \mathrm{s}^{-1}$ (STP$1003 \mathrm{C}$, Edwards, USA), respectively. The vacuums of doping and detection chambers are $2.2 \times 10^{-6}$ and $1 \times 10^{-6} \mathrm{~Pa}$ without the beam, respectively. The droplets source consists of an assembly holding a cryogenic Even-Lavie valve (EL-C-C-2009, Israel), which is thermally connected to the cold head of a closed- cycle refrigerator system (RDK-415E, Sumitomo Heavy Industries, Japan). The temperature of the nozzle can be adjusted with an accuracy of $0.5 \mathrm{~K}$ by heating the assembly by a 25 $\mathrm{W}$ cartridge heater which is powered by a temperature controller (TC202, East Changing, China). The droplets beam is formed by expanding high purity helium gas $\left({ }^{4} \mathrm{He}, 99.9999 \%\right)$ with a stagnation pressure of $3.0 \times 10^{6} \mathrm{~Pa}$ from the pulsed valve whose temperatures were kept in the range of $16-24 \mathrm{~K}$. The expanding gas accelerates and cools adiabatically. Then the helium clusters beam is formed and collimated by a conical skimmer (Beam Dynamics) with a diameter of $2 \mathrm{~mm}$ which is placed at a distance of $15 \mathrm{~cm}$ from the source. In such conditions, the mean droplet radius ranges from $1.7 \mathrm{~nm}$ (consisting of 500 helium atoms on average) to $5.1 \mathrm{~nm}$ (consisting of 12740 helium atoms on average). Passing through the skimmer, the droplets beam enters the doping chamber and picks up impurities via collision. The doping sample is introduced into the chamber through a leak valve (LVM940, VG Scienta, Sweden) which is continuously controllable between $10^{-1}$ and $10^{-9} \mathrm{~Pa} \cdot \mathrm{s}^{-1}$. The length of the doping chamber is $8 \mathrm{~cm}$. By adjusting the leak rate the constant scattering gas pressure is obtained. The doping chamber pressure is optimized at $4.6 \times 10^{-3} \mathrm{~Pa}$ to maximize the pickup of a single OCS molecule. The doped droplets enter the detection chamber by passing through the other $2 \mathrm{~mm}$ skimmer (Beam Dynamics, USA). The photodissociation and ionization laser interact with the doped droplets beam perpendicularly at the center of the VMI spectrometer. The doped beam, laser, and VMI spectrometer are orthogonal in the detection chamber.

In terms of the photodissociation of OCS inside helium droplets, the excitation wavelengths $(230.4-229.8 \mathrm{~nm})$ were provided by a Nd:YAG (Continuum PL DLS 8000) pump tunable dye laser (Sirah CBST-G-18) with Coumarin 460 dye. The frequency-doubled laser beam ( $230 \mathrm{~nm}$, typical energy $\sim 0.5-1.5 \mathrm{~mJ}$ • pulse $\left.^{-1}\right)$ was focused with a len $(f=250 \mathrm{~mm})$ onto the helium droplet beam. The $\mathrm{CO}^{+}$fragment ions formed in the subsequent ionization were accelerated by a set of well adjusted ion optics assembly and detected by a Chevron-type dual MCP's coupled to a P-47 phosphor screen (Burle Electro-Optics). A negative high voltage pulse generator (PVM-4210, DEI; typical duration $\sim 200 \mathrm{~ns}$ ) was used for mass selection. The VMI images 
from the phosphor screen were recorded by a digital camera (scA780-54gm) and further transferred to a computer. The total fluorescence from the phosphor screen was monitored by a photomultiplier tube (PMT). The timing of the pulse valve, the laser, and the gate pulse applied on the MCP's was controlled by a multichannel digital delay pulse generator (BNC Model $555)$.

\section{Results and discussion}

The $(2+1)$ REMPI spectra $(229.8-230.3 \mathrm{~nm} ; Q$ branch of the $B^{1} \Sigma^{+} \longleftarrow X^{1} \Sigma^{+}$transition $^{17}$ ) of $\mathrm{CO}$ fragments dissociated from OCS molecules are shown in Fig.1. The solid line and dotted line show two different conditions: (1) effusive beam of OCS molecules in the gas phase; (2) a single OCS molecule embedded in helium droplets, respectively. For case (1), the spectral features related to $\mathrm{CO}(v=0)$ and $\mathrm{CO}(v=1)$ all exhibit high $J$ and bimodal distributions. ${ }^{17,23}$ Previous studies confirmed that the bimodal distribution is mainly due to the different dissociation pathways on the $2^{1} A^{\prime}\left({ }^{1} \Delta\right)$ potential energy surface, named direct dissociation and internal conversion to the ground state. ${ }^{12,24,25}$ For case (2), however, rotational distributions for both $\mathrm{CO}(v=0)$ and $\mathrm{CO}(v=1)$ all go to low- $J$ 's while the high- $J$ components disappear, as shown in the superimposed assignment in Fig.1. This indicates that in the helium droplets environment rotational cooling is much more efficient than vibrational cooling, as also observed in other molecular systems.

In order to obtain the insight into the dissociation dynamics, we chose the intense band-heads of $\mathrm{CO}(v=0,1)$ and recorded the images from the photodissociation of OCS doped helium droplets. The typical image recorded at $2.8 \mathrm{~nm}$ droplets size is shown in Fig.2(a). Due to the sensitive REMPI probe of CO molecule, the $\mathrm{CO}$ ions from the effusive OCS beam photodissociation and thermal background in the vacuum chamber were also accumulated by the imaging detection. However, benefited from the velocity mapping technique,${ }^{26}$ the background signal was separated very well spatially and could be subtracted

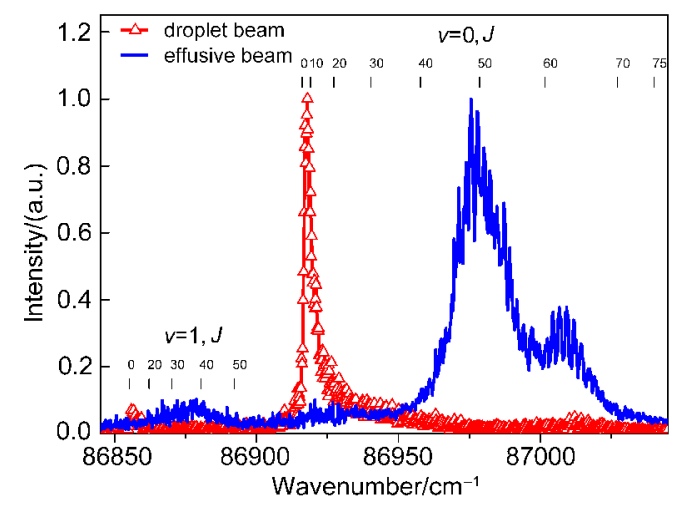

Fig.1 (2+1) REMPI spectra ( $Q$ branch of the $B^{1} \Sigma^{+} \longleftarrow X^{1} \Sigma^{+}$ transition) of CO from photodissociation of OCS embeded helium droplets at $\sim 230 \mathbf{n m}$

The rotational assignments of $\mathrm{CO}$ products are superimposed in the figure. accordingly. As shown in Fig.2(a) the region in the circle is the interested CO signal from the photodissociation of OCS doped helium droplets. The speed and angular distributions can be derived from the raw image with the BASEX method ${ }^{27}$ and be corrected for the homogenous image background noise using the procedure described previously. ${ }^{28}$ We accumulated several images with different droplets sizes. All angular distributions from resulting images are nearly isotropic. However, some intriguing speed distributions for $\mathrm{CO}(v=0)$ are observed, as shown in Fig.2(b). Obviously, the speed distributions (peaking around $240 \mathrm{~m} \cdot \mathrm{s}^{-1}$ ) for the photodissociation of doped helium droplets are much lower than those from the isolated gaseous OCS molecule dissociation (peaking at $1350 \mathrm{~m} \cdot \mathrm{s}^{-1}$ ). Previous studies on alkyl iodides doped helium droplets dissociation ${ }^{6-8}$ imply that the "direct" mechanism could dominate the process.

The photofragments may push away helium atoms, travel through the confined superfluid system, and depart into the gas phase. Due to the high speed of the photofragements, which is largely exceed the Landau critical velocity $\left(58 \mathrm{~m} \cdot \mathrm{s}^{-1}\right)$ in He II, the escaping process should overcome the friction and lose the kinetic energy to superfluid environment. The results show that the relaxation of the speed and angular distributions is strongly mass dependent. Briefly, the lighter photofragments lose more kinetic energy and have the larger reduction of the anisotropy. More than $95 \%$ of the kinetic energies are relaxed for $\mathrm{CH}_{3}$ and $\mathrm{C}_{2} \mathrm{H}_{5}$ products. But the value shifts to $70 \%$ for escaping iodine atoms. For the $\mathrm{CO}$ products probed in the present studies, de-

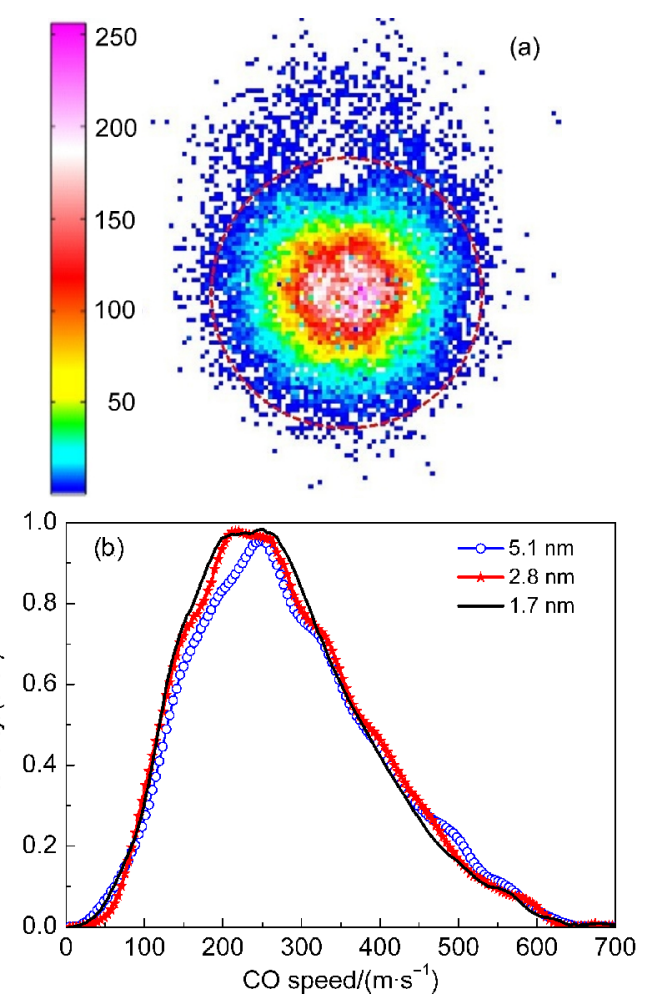

Fig.2 (a) Typical velocity map image of $\mathrm{CO}(v=0)$ resulting from the photodissociation of OCS embeded helium droplets at an average size of $2.8 \mathrm{~nm}$; (b) $\mathrm{CO}(v=0)$ speed distributions for the selected different sizes of helium droplets 
tailed survey shows that average $\sim 97 \%$ of the kinetic energies transfer to the helium environment, which agrees very well with the previous studies. The average kinetic energies for the dissociation of the different sizes of the doped helium droplets are listed in Table 1. The dependence of the average kinetic energies with helium droplet sizes shows somehow contrary of the previous observations. Previous direct mechanism implies that the average kinetic energy distributions shift to lower with increasing the droplet sizes. However, the kinetic energy distributions derived from ground state of $\mathrm{CO}$ images show that the average kinetic energies are increased with the increase of the droplets sizes. We proposed a mechanism for the intriguing findings as follows. The OCS parent molecules embedded in helium droplets are photodissociated into $\mathrm{CO}$ and $\mathrm{S}$ fragments. The CO fragments lose kinetic as well as rotational energies by colliding with the surrounding helium atoms, and subsequently the rotationally cooled CO fragments are ionized by REMPI laser inside the helium droplets. The charged $\mathrm{CO}^{+}$fragment in droplets will transfer energy to the helium atoms to form a local high-pressure gas bubble, ${ }^{29}$ and the bubble will inevitably expand to the surface of droplet. The external electric field will accelerate such an expansion. ${ }^{30}$ When the bubble moves to the surface of droplet, $\mathrm{CO}^{+}$escapes from the droplet to vacuum as a bare ion. According to the law of momentum/energy conservation, the $\mathrm{CO}^{+}$fragment will acquire higher speed distribution with increasing the droplet size (i.e., decreasing the mass ratio of CO over helium droplet), as the available energy is kept constant. Besides the electron bubble, the dimple generated on droplets surface may contribute to the trend of the average kinetic energy distributions. As described previously, the alkali atoms and clusters interact weakly with the helium and reside in a dimple on the helium droplets surface. ${ }^{31-34}$ Considering the photodissociation of OCS doped helium droplets, the CO fragments move to the droplet surface and stay in the dimple within several picoseconds. This exit time of photofragment is much shorter than the laser pulse width ( $8 \mathrm{~ns})$. Therefore considerable amount of the $\mathrm{CO}$ fragments reside in the dimples and are ionized on the surface which produces the two-body alike dissociation phenomenon.

We also extended studies to the vibrationally excited product probe. The images of $\mathrm{CO}(v=1)$ were recorded with droplet sizes of 1.7, 2.8, and $5.1 \mathrm{~nm}$, respectively. Again, all these images show nearly isotropic angular distributions. The speed distributions of $\mathrm{CO}(v=1)$ fragments are shown in Fig.3. Obviously, the average kinetic energies for products $(v=1)$ are larger than

Table 1 Average translational energies of $\mathrm{CO}$ products from photodissociation of OCS doped helium droplets with various mean droplet sizes

\begin{tabular}{ccc}
\hline Droplet size $/ \mathrm{nm}$ & $\left\langle E_{\mathrm{CO}(\mathrm{v}-0)}>/ \mathrm{cm}^{-1}\right.$ & $\left\langle E_{\mathrm{CO}(\mathrm{v}-1)}>/ \mathrm{cm}^{-1}\right.$ \\
\hline 5.1 & 75.2 & 91.1 \\
2.8 & 74.0 & 103.2 \\
1.7 & 70.4 & 96.0 \\
\hline
\end{tabular}

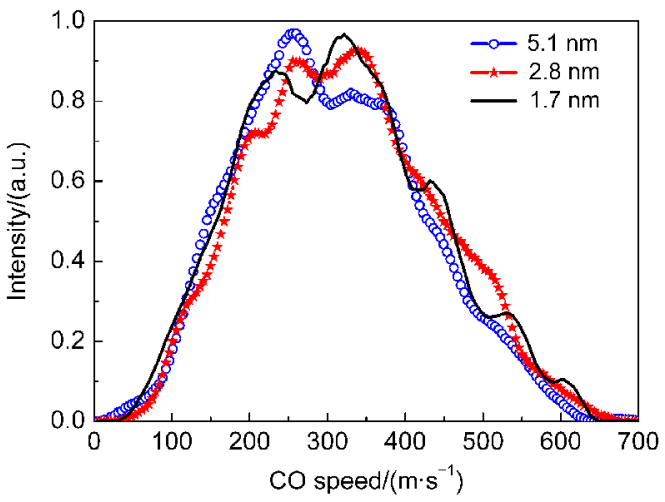

Fig.3 CO $(v=1)$ speed distributions for the photodissociation of OCS doped helium droplets at selected average sizes

those for fragments $(v=0)$, as show in Fig. 3 and Table 1. The results are contrary to the gaseous phase observations. Considering the same available energy, products $(v=1)$ take more energy for internal excitation. The less kinetic energy releases are expected for the vibrationally excited products. We refer to assign this to the difference of the interaction of the finite-sized superfluid helium with the vibrational ground state and vibrationally excited state products. As discussed above, the "direct" nonthermal process dominates the generation process of vibrational ground state products. However, the vibrationally excited products may be governed by the ion ejection model. More related to this issue, we recall the IR spectra of cations embedded in superfluid helium droplets. Drabbels et al..$^{35,36}$ have developed a spectroscopic method to study the IR spectra of the cold cations inside the helium droplets. The experimental evidence indicates that after excitation the embedded ions are ejected from the droplets which are governed by a nonevaporative process. The kinetic energy of the departing ions is molecular specific and independence of the excitation energy. Their studies on 1,1-diphenylethylene (DPE) cations show that the different vibrational excitations of the embedded cations have the identical speed distributions. Qualitatively, the present $\mathrm{CO}(v=1)$ results agree with the previous DPE studies. Both results provide a new insight of cation ejection from embedded helium droplets, the understanding of which invokes further ongoing experiments and theoretical calculations.

\section{Conclusions}

To summarize, we have studied photodissociation dynamics of OCS molecules embedded in helium droplets using REMPI combined with velocity map imaging techniques. Rotational cooling is found to be much more efficient than vibrational cooling in helium droplets. Nearly isotropic angular distributions were observed in the velocity map images for both $\mathrm{CO}$ $(v=0)$ and CO $(v=1)$. The slightly modified "direct" mechanism is proposed for the $\mathrm{CO}(v=0)$ fragments departing from the droplets. For the case of $\mathrm{CO}(v=1)$, the non-evaporative ion ejection process is confirmed. 


\section{References}

(1) Toennies, J. P.; Vilesov, A. F. Angew. Chem. Int. Edit. 2004, 43, 2622.

(2) Grebenev, S.; Hartmann, M.; Havenith, M.; Sartakov, B.; Toennies, J. P.; Vilesov, A. F. J. Chem. Phys. 2000, 112, 4485. doi: 10.1063/1.481011

(3) Hartmann, M.; Pörtner, N.; Sartakov, B.; Toennies, J. P.; Vilesov, A. F. J. Chem. Phys. 1999, 110, 5109. doi: 10.1063/ 1.479111

(4) Choi, M. Y.; Douberly, G. E.; Falconer, T. M.; Lewis, W. K.; Lindsay, C. M.; Merritt, J. M.; Stiles, P. L.; Miller, R. E. Int Rev. Phys. Chem. 2006, 25, 15. doi: 10.1080/ 01442350600625092

(5) Toennies, J. P.; Vilesov, A. F. Annu. Rev. Phys. Chem. 1998, 49, 1. doi: 10.1146/annurev.physchem.49.1.1

(6) Braun, A.; Drabbels, M. J. Chem. Phys. 2007, 127, 114305. doi: $10.1063 / 1.2767263$

(7) Braun, A.; Drabbels, M. J. Chem. Phys. 2007, 127, 114303. doi: $10.1063 / 1.2767261$

(8) Braun, A.; Drabbels, M. J. Chem. Phys. 2007, 127, 114304. doi: $10.1063 / 1.2767262$

(9) Pentlehner, D.; Nielsen, J. H.; Slenczka, A.; Mølmer, K.; Stapelfeldt, H. Phys. Rev. Lett. 2013, 110, 093002. doi: 10.1103/ PhysRevLett.110.093002

(10) Sato, Y.; Matsumi, Y.; Kawasaki, M.; Tsukiyama, K.; Bersohn, R. J. Phys. Chem. 1995, 99, 16307. doi: 10.1021/j100044a017

(11) Brouard, M.; Quadrini, F.; Vallance, C. J. Chem. Phys. 2007, 127, 084305. doi: 10.1063/1.2757619

(12) Suzuki, T.; Katayanagi, H.; Nanbu, S.; Aoyagi, M. J. Chem. Phys. 1998, 109, 5778. doi: 10.1063/1.477200

(13) Sugita, A.; Mashino, M.; Kawasaki, M.; Matsumi, Y.; Bersohn, R.; Trott-Kriegeskorte, G.; Gericke, K. H. J. Chem. Phys. 2000, 112, 7095. doi: 10.1063/1.481324

(14) Kim, M. H.; Li, W.; Lee, S. K.; Suits, A. G. Can. J. Chem. 2004, 82, 880. doi: $10.1139 / \mathrm{v} 04-072$

(15) Rakitzis, T. P. Science 2004, 303, 1852. doi: 10.1126/ science. 1094186

(16) Brouard, M.; Green, A. V.; Quadrini, F.; Vallance, C. J. Chem. Phys. 2007, 127, 084304. doi: 10.1063/1.2757618

(17) Rijs, A. M.; Backus, E. H. G.; de Lange, C. A.; Janssen, M. H. M.; Westwood, N. P. C.; Wang, K.; McKoy, V. J. Chem. Phys. 2002, 116, 2776. doi: 10.1063/1.1434993
(18) Katayanagi, H.; Suzuki, T. Chem. Phys. Lett. 2002, 360, 104. doi: 10.1016/S0009-2614(02)00788-1

(19) Grebenev, S.; Havenith, M.; Madeja, F.; Toennies, J. P.; Vilesov, A. F. J. Chem. Phys. 2000, 113, 9060. doi: 10.1063/1.1286243

(20) Kunze, M.; Markwick, P. R. L.; Pörtner, N.; Reuss, J.; Havenith, M. J. Chem. Phys. 2002, 116, 7473. doi: 10.1063/1.1467330

(21) Grebenev, S. Science 1998, 279, 2083. doi: 10.1126/ science. 279.5359 .2083

(22) Zhang, C.; Zhang, Z.; Huang, C.; Zhang, Q.; Chen, Y. Chin. J. Chem. Phys. 2013, 26, 270. doi: 10.1063/1674-0068/26103/270276

(23) van den Brom, A. J.; Rakitzis, T. P.; van Heyst, J.; Kitsopoulos, T. N.; Jezowski, S. R.; Janssen, M. H. M. J. Chem. Phys. 2002, 117, 4255. doi: 10.1063/1.1496464

(24) Mo, Y. X.; Katayanagi, H.; Heaven, M. C.; Suzuki, T. Phys. Rev Lett. 1996, 77, 830. doi: 10.1103/PhysRevLett.77.830

(25) Katayanagi, H.; Mo, Y. X.; Suzuki, T. Chem. Phys. Lett. 1995, 247, 571. doi: 10.1016/S0009-2614(95)01253-2

(26) Eppink, A. T. J. B.; Parker, D. H. Rev. Sci. Instrum. 1997, 68, 3477. doi: $10.1063 / 1.1148310$

(27) Dribinski, V.; Ossadtchi, A.; Mandelshtam, V. A.; Reisler, H. Rev. Sci. Instrum. 2002, 73, 2634. doi: 10.1063/1.1482156

(28) Braun, A.; Drabbels, M. Rev. Sci. Instrum. 2005, 76, 113103. doi: $10.1063 / 1.2130941$

(29) Lewis, W. K.; Applegate, B. E.; Sztáray, J.; Sztáray, B.; Baer, T.; Bemish, R. J.; Miller, R. E. J. Am. Chem. Soc. 2004, 126, 11283. doi: $10.1021 / \mathrm{ja} 030653 \mathrm{q}$

(30) Fárnšk, M.; Henne, U.; Samelin, B.; Toennies, J. P. Phys. Rev. Lett. 1998, 81, 3892. doi: 10.1103/PhysRevLett.81.3892

(31) Loginov, E.; Callegari, C.; Ancilotto, F.; Drabbels, M. J. Phys. Chem. A 2011, 115, 6779. doi: 10.1021/jp111146n

(32) Stienkemeier, F.; Higgins, J.; Ernst, W.; Scoles, G. Phys. Rev. Lett. 1995, 74, 3592. doi: 10.1103/PhysRevLett.74.3592

(33) Ancilotto, F.; Lerner, P. B.; Cole, M. W. J. Low Temp. Phys. 1995, 101, 1123. doi: 10.1007/BF00754527

(34) Stienkemeier, F.; Higgins, J.; Ernst, W. E.; Scoles, G. Z Physica $B$ 1995, 98, 413. doi: 10.1007/BF01338416

(35) Smolarek, S.; Brauer, N. B.; Buma, W. J.; Drabbels, M. J. Am. Chem. Soc. 2010, 132, 14086. doi: 10.1021/ja1034655

(36) Zhang, X.; Brauer, N. B.; Berden, G.; Rijs, A. M.; Drabbels, M. J. Chem. Phys. 2012, 136, 044305. doi: 10.1063/1.3678011 